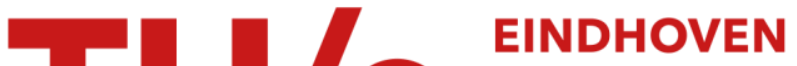 \\ UNIVERSITY OF \\ TECHNOLOGY
}

\section{Modeling of burner-stabilized hydrogen/air flames using mathematically reduced reaction schemes}

\section{Citation for published version (APA):}

Eggels, R. L. G. M., \& Goey, de, L. P. H. (1995). Modeling of burner-stabilized hydrogen/air flames using mathematically reduced reaction schemes. Combustion Science and Technology, 107(1-3), 165-180. https://doi.org/10.1080/00102209508907800

DOI:

10.1080/00102209508907800

Document status and date:

Published: 01/01/1995

\section{Document Version:}

Publisher's PDF, also known as Version of Record (includes final page, issue and volume numbers)

\section{Please check the document version of this publication:}

- A submitted manuscript is the version of the article upon submission and before peer-review. There can be important differences between the submitted version and the official published version of record. People interested in the research are advised to contact the author for the final version of the publication, or visit the $\mathrm{DOI}$ to the publisher's website.

- The final author version and the galley proof are versions of the publication after peer review.

- The final published version features the final layout of the paper including the volume, issue and page numbers.

Link to publication

\section{General rights}

Copyright and moral rights for the publications made accessible in the public portal are retained by the authors and/or other copyright owners and it is a condition of accessing publications that users recognise and abide by the legal requirements associated with these rights.

- Users may download and print one copy of any publication from the public portal for the purpose of private study or research.

- You may not further distribute the material or use it for any profit-making activity or commercial gain

- You may freely distribute the URL identifying the publication in the public portal.

If the publication is distributed under the terms of Article 25fa of the Dutch Copyright Act, indicated by the "Taverne" license above, please follow below link for the End User Agreement:

www.tue.nl/taverne

Take down policy

If you believe that this document breaches copyright please contact us at:

openaccess@tue.nl

providing details and we will investigate your claim. 


\title{
Modeling of Burner-Stabilized Hydrogen/Air Flames Using Mathematically Reduced Reaction Schemes
}

\author{
R. L. G. M. EGGLES and L. P. H. De GOEY Eindhoven University of Technology, \\ Faculty of Mechanical Engineering (WOC), PO Box 513, 5600 MB Eindhoven, \\ The Netherlands
}

(Received November 15, 1994; in final form April 20,1995)

\begin{abstract}
A mathematical technique is used to reduce several hydrogen/air reaction systems to oneand two-step schemes. The reduction technique is based on the use of intrinsic low-dimensional manifolds in composition space as introduced by Maas and Pope (1992). In this method it is assumed that the fastest reaction groups of the chemical source term are in steady-state.

For a reaction mechanism that does not include $\mathrm{HO}_{2}$, a one-step reduced scheme is used for burnerstabilized hydrogen/air flame calculations. It appears that the one-step reduced scheme predicts the flame structure quite well for several values of the equivalence ratio and mass flow rates. The differences in flame temperature between the reduced scheme and full scheme calculations are less than $50 \mathrm{~K}$.

A one-step reduced scheme is also used for the reaction scheme including $\mathrm{HO}_{2}$. For this scheme, however, only low mass flow rates can be used, otherwise the flame will blow off. This is caused by the fact that the one-step scheme underestimates the adiabatic burning velocity considerably (Eggels, 1995). However, the one-step reduced scheme still predicts the main species quite well. For larger mass flow rates, close to the adiabatic mass burning rate, a two-step reduced scheme is used instead. The two-step scheme gives a significant improvement of the $\mathrm{H}_{2}$ /air flame structure, as expected.
\end{abstract}

Key Words: Laminar flames, reduced reaction mechanisms

\section{INTRODUCTION}

To describe combustion phenomena in more-dimensional combustion geometries, the use of complex chemistry leads to excessive computational effort. On the other hand, accurate chemical information is often required e.g., for the prediction of the NO emission. A large part of the combustion research today is therefore based on the application of reduced reaction mechanisms. We can identify different approaches to reduce a reaction mechanism. The conventional technique is based on steady-state assumptions for intermediate species and partial-equilibrium assumptions for certain reactions (Peters, 1987, 1993) (Smooke, 1991). The strategy applied to find out which species and reactions may be taken in steady-state or partial-equilibrium requires much understanding of chemical kinetics. Another method to simplify chemical kinetics is to decouple the reaction system into fast and slow reaction groups. These reaction groups, which are linear combinations of elementary reactions, can be obtained automatically, so that less insight in chemical kinetics is required. When the fast and slow reaction groups are known, different approaches can be followed. Lam (1988) and Goussis (1992) use this information to find out which steady-state assumptions for the species and partial-equilibrium assumptions for a set of elementary reactions can be applied 
locally. This CSP (Computational Singular Perturbation) method can be used to solve the full set of differential equations of the complex reaction system fast and accurately. The knowledge of reaction groups with different time scales can also be used by applying partial-equilibrium assumptions for the fastest reaction groups (Maas, 1992). The difference with the previously mentioned conventional reduction technique is that it is not necessary to know the relationship between the reaction groups and elementary reactions. It has been shown (Maas, 1992, 1994), (Eggels, 1995) that this method gives appropriate results if it is used for a perfectly stirred reactor and flame calculations. The computational effort for these reduced calculations is much smaller than for the full scheme calculations. Another major difference with other reduction methods is that the solution method is separated into two parts. The low-dimensional manifold in composition space, defined by the steady-state equations, is parameterized by using progress or controlling variables and is calculated first. This implies that in the second step, the actual flame calculations, only the differential equations for the controlling variables have to be solved by using the manifold, in form of look-up tables, for the other species.

We use this method to reduce the hydrogen/air reaction mechanism to one- and two-step reduced schemes. Previous investigations (Eggels, 1995) have shown that a hydrogen/air system without $\mathrm{HO}_{2}$ species, reduced to a one- step reduced scheme, gives accurate results for adiabatic flat flames. If $\mathrm{HO}_{2}$ is included, the time scale of the slowest process that is supposed to be in steady-state is of the same order of magnitude as the time scale that describes the time evolution on the manifold, for relatively low temperatures. The specific mole number of $\mathrm{HO}_{2}$ reaches its maximum in this region. Because the chemical source term and also the burning velocity is sensitive to variations in $\mathrm{HO}_{2}$ specific mole numbers, this scheme does not give appropriate results.

In this paper we reduce $\mathrm{H}_{2}$ /air reaction schemes, with and without $\mathrm{HO}_{2}$, to a one-step mechanism. As expected, it is not possible to model burner-stabilized flames for flow velocities close to the adiabatic burning velocity with the one-step reduced scheme when the reaction mechanism including $\mathrm{HO}_{2}$ is applied. This problem is solved by the application of a two-step reduced scheme, for large flow velocities. The method to reduce a reaction mechanism is explained briefly in the next section. We come to the use of the low-dimensional manifolds for the flat flame calculations in the subsequent section. The results are compared with full scheme calculations and discussed in the last section, finally.

\section{MATHEMATICALLY REDUCED MECHANISMS}

The mathematical reduction technique, introduced by Maas and Pope (1992), is based on the assumption that fast reaction groups in the combustion process are in steadystate. The fast and slow reaction groups are separated with the use of an eigenvector analysis of the Jacobian matrix of the chemical source term (Maas, 1992) (Lam, 1988) (Eggels, 1995). We suppose that convective and diffusive transport processes proceed much slower than the fastest reaction groups. To study the dynamical behaviour of the reaction mechanism, diffusive and convective terms are omitted. The effect of convec- 
tion and diffusion is considered later on in this paper. Therefore, we consider the following system:

$$
\frac{\partial \phi_{i}}{\partial t}=w_{i},
$$

where $\phi_{i}$ denotes the specific mole number of species $i$. The specific mole number is defined as the mass fraction of the species $Y_{i}$ divided by its molar mass $M_{i}$. The system is described in composition space by writing the composition in vector notation: $\boldsymbol{\phi}=\left(\phi_{1}, \ldots, \phi_{n}\right)^{T}$, where $n$ denotes the number of species. Equation (1) is linearized around a reference composition $\phi^{0}$ :

$$
\boldsymbol{w}(\phi)=\boldsymbol{w}\left(\phi^{0}\right)+\frac{\partial \boldsymbol{w}\left(\phi^{0}\right)}{\partial \phi}\left(\phi-\phi^{0}\right) .
$$

When Equation (1) is transformed to the basis of eigenvectors, using $\boldsymbol{\phi}=\boldsymbol{S} \boldsymbol{\phi}^{\prime}$ (the coordinates in the eigenvector basis are denoted with an accent), the differential equations are decoupled:

$$
\frac{\partial \boldsymbol{\phi}^{\prime}}{\partial t}=\boldsymbol{w}^{\prime}\left(\boldsymbol{\phi}^{0}\right)+\boldsymbol{\Lambda}\left(\boldsymbol{\phi}^{\prime}-\boldsymbol{\phi}^{\prime}\right),
$$

where $\boldsymbol{A}$ is the diagonal matrix of eigenvalues. The solutions of these decoupled equations are found easily and are given by:

$$
\phi_{i}^{\prime}=\phi_{i}^{\prime 0}+\frac{w_{i}^{\prime}\left(\phi^{0}\right)}{\lambda_{i}}\left(\exp \left(\lambda_{i} t\right)-1\right) .
$$

Equation (4) indicates that the typical time scales are

$$
\tau_{i}=1 /\left|\mathscr{R} e\left(\lambda_{i}\right)\right|,
$$

where $\mathscr{R} e\left(\lambda_{i}\right)$ denotes the real part of eigenvalue $i$. Ordering of the eigenvalues in descending order of real parts $\left(\mathscr{R} e\left(\lambda_{1}\right) \geq \cdots \geq \mathscr{R} e\left(\lambda_{n}\right)\right)$, provides that the fastest reaction groups $\phi_{i}^{\prime}$ with negative real parts are those with the highest index $i$. Note, that reaction groups which correspond with eigenvalues with positive real parts can not be assumed in steady-state. The steady-state assumptions for the fastest reaction groups are given by:

$$
\frac{\partial \phi_{i}^{\prime}}{\partial t}=0, \quad i=n_{c}+n_{e}+1, \ldots, n .
$$

The number of reaction groups that has to be taken in steady-state depends on the desired dimension of the reduced scheme $n_{c}$ and is equal to $n-n_{c}-n_{e}$, where $n_{e}$ is the number of elements in the system.

To obtain the steady-state assumptions in the original basis we transform Equation (6) by using $\phi^{\prime}=\boldsymbol{S}^{-1} \phi$, where $\boldsymbol{S}^{-1}$ denotes the transformation matrix given by:

$$
S^{-1}=\left(\begin{array}{ccc}
- & s_{1}^{L} & - \\
& \vdots & \\
- & s_{n}^{L} & -
\end{array}\right),
$$


where $s_{i}^{L}$ are the left eigenvectors of the Jacobian matrix. This yields:

$$
\left(s_{i}^{L}, \frac{\partial \phi}{\partial t}\right)=0, \quad i=n_{c}+n_{e}+1, \ldots, n .
$$

Substitution of Equation (1) gives finally:

$$
\left(s_{i}^{L}, w\right)=0, \quad i=n_{c}+n_{e}+1, \ldots, n .
$$

These equations define a low-dimensional manifold in composition space. Processes in this subspace proceed slower than processes outside the manifold.

Since the steady-state Equations (9) depend on the local composition of the mixture it would cost too much computational effort to solve the steady-state relations during the application of the reduced scheme in flame computations. The Equations (9) are therefore solved beforehand instead, by making a parameterization of the manifold in the form of controlling variables, which describe the progress of the process on the manifold. Linear combinations of the specific mole numbers $\alpha_{i}=\left(\xi_{i}, \phi\right)$ may be chosen as controlling variables, as long as there exists only one manifold composition for each combination of the controlling variables.

It has to be kept in mind that the specific element mole numbers, enthalpy and pressure appear as additional degree of freedom on the manifold as these may change by transport processes in the flame. The specific element mole numbers, denoted by $\chi_{i}$ are given by $\chi_{i}=\left(\boldsymbol{\mu}_{i}, \boldsymbol{\phi}\right)$, where $\boldsymbol{\mu}_{i}$ denotes the composition vector of element $i\left(\mu_{j, i}\right.$ is the number of atoms of element $i$ in species $j$ ). These specific element mole numbers are not changed by chemical reactions as they are equal to $z_{i} / W_{i}$, with $z_{i}$ the mass fraction and $W_{i}$ the molar mass of element $i$. In general, the number of degrees of freedom of the manifold is equal to $n_{c}+n_{e}+2$. The manifold composition is calculated for all physically reasonable values of the above introduced parameters. The set of equations that has to be solved to calculate a point on the manifold for controlling variables $\alpha_{1} \cdots \alpha_{n_{e}}$, specific element mole numbers $\chi_{1} \cdots \chi_{n_{e}}$, enthalpy $h_{\text {ref }}$ and pressure $p_{\text {ref }}$ is given by $g=\mathbf{o}$, denoting:

$$
\begin{array}{ll}
g_{i}=\left(\xi_{i}, \boldsymbol{\phi}\right)-\alpha_{i}=0, & i=1, \ldots, n_{c}, \\
g_{i}=\left(\boldsymbol{\mu}_{\left(i-n_{c}\right)}, \boldsymbol{\phi}\right)-\chi_{\left(i-n_{c}\right)}=0, & i=n_{c}+1, \ldots, n_{c}+n_{e}, \\
g_{i}=\left(\boldsymbol{s}_{i}^{L}, \boldsymbol{w}\right)=0 . & i=n_{c}+n_{e}+1, \ldots, n, \\
g_{n+1}=\sum_{i=1}^{n} M_{i} \phi_{i}\left(h_{i}^{0}+\int_{T_{0}}^{T} c_{p_{i}} \tau d \tau\right)-h_{\mathrm{ref}}, & \\
g_{n+2}=p-p_{\text {ref }} . &
\end{array}
$$

To obtain the specific mole numbers and temperature in a single manifold point, the Equation set (10) is solved by using Newton's method, which can be extended with a pseudo time-stepping method to enlarge the convergence region if necessary.

Up to here we only considered a time-dependent system. In this paper, however, we study burner-stabilized flat flames. For these flames, the velocities.are much lower than the speed of sound so that the pressure may be assumed to be constant. In this paper we will use unit Lewis numbers for simplicity. It can be shown that the specific element 
mole numbers remain frozen and disappear as degree of freedom on the manifold. Hence, the number of degrees of freedom reduces to $n_{c}+1$. We have to emphasize that the use of unit Lewis numbers is not physically realistic. The main objective of this paper, however, is to investigate the performance of the chemical reduction technique. For the comparison of reduced and complex computations, the transport model is therefore of minor interest. To make a fair comparison unit Lewis numbers are used for the reduced as well as for the complex computations. For the flat flames we have to take convection and diffusion into consideration. How this is done, is explained in the next section.

\section{BURNER STABILIZED FLAMES}

The transport equations for the species in stationary flat flames are given by:

$$
\dot{M} \frac{d \phi_{i}}{d x}-\frac{d}{d x}\left(\rho D_{i} \frac{d \phi_{i}}{d x}\right)=\rho w_{i} \quad \text { for } i=1, \ldots, n
$$

where $\dot{M}=\rho u$ denotes the constant mass flow rate. Apart from these conservation equations for the components we have to take the enthalpy equation into consideration.

For the reduced scheme only $n_{c}$ differential equations for the controlling variables and one for the enthalpy have to be solved. How the differential equations for the controlling variables are obtained from the original equations for the species is explained in this section. This is done in two steps. First, the composition is described by a reduced number of variables. The differential equations of the species are projected on the manifold, subsequently.

To describe the evolution of the process on the manifold, we substitute $\boldsymbol{\phi}=\boldsymbol{\phi}\left(\alpha_{1}, \ldots, \alpha_{n_{c}}, h\right)$ in Equation(11). For the derivatives $d \phi_{i} / d x$ we may write:

$$
\frac{d \phi_{i}}{d x}=\sum_{j=1}^{n_{c}} \frac{\partial \phi_{i}}{\partial \alpha_{j}} \frac{\partial \alpha_{j}}{\partial x}+\frac{\partial \phi_{i}}{\partial h} \frac{\partial h}{\partial x}
$$

As we consider a one-dimensional system, all variables depend on the spatial coordinate $x$ only. Suppose that a suitable controlling variable $\alpha_{j}$ increases or decreases monotonously with $x$. Then, all variables may be considered as a function of $\alpha_{j}$ also. This means that we may write $\partial \alpha_{i} / \partial x=\left(\partial \alpha_{i} / \partial \alpha_{j}\right)\left(\partial \alpha_{j} / \partial x\right)$ and $\partial h / \partial x=\left(\partial h / \partial \alpha_{j}\right)\left(\partial \alpha_{j} / \partial x\right)$. Substitution of this into (12) gives:

$$
\frac{d \phi_{i}}{d x}=\frac{d \phi_{i}}{d \alpha_{j}} \frac{d \alpha_{j}}{d x}
$$

Note that we have to use monotonously increasing or decreasing controlling variables, otherwise $d \phi_{i} / d \alpha_{j}$ could be zero.

For the differential Equation (11) of the reduced scheme we now obtain:

$$
\dot{M} \frac{d \phi_{i}}{d \alpha_{j}} \frac{d \alpha_{j}}{d x}-\frac{d \rho D_{i}}{d \alpha_{j}} \frac{d \phi_{i}}{d \alpha_{j}}\left(\frac{d \alpha_{j}}{d x}\right)^{2}-\rho D_{i}\left(\frac{d^{2} \phi_{i}}{d \alpha_{j}^{2}}\left(\frac{d \alpha_{j}}{d x}\right)^{2}+\frac{d \phi_{i}}{d \alpha_{j}} \frac{d^{2} \alpha_{j}}{d x^{2}}\right)-\rho w_{i}=0
$$


As Equation (14) yields for all $i$, we have $n$ equations and only $n_{c}$ independent variables $\alpha_{i}$. This is not a contradiction, because variations of the compositions are restricted; the composition has to be on the manifold. Therefore, Equations (14) are projected on the manifold. In Appendix A we will show that projection of the convection term and the chemical source term are identical to the convection term and source term of the original species equation for the controlling variables (11). From a physical point of view it can be understood that the convection and chemical source term vectors are already in the tangent plane of the manifold, as observed by Maas and Pope (1994). The projection of the diffusion term, however, is not equal to the diffusion term of the initial differential equation for the controlling variable. To project the diffusion term on the manifold we can use a projection method based on the tangential direction vectors of the manifold as well a projection method based on the eigenvectors. Both projection methods are described and explained in more detail in Appendix A. Here, we used the projection method based on the tangential direction vectors, so that we don't have to store eigenvectors. Furthermore, in Appendix A it is shown that differences between the projection methods are small. The differential equation reads finally:

$$
\begin{aligned}
& \dot{M} \frac{d \alpha_{j}}{d x}-\sum_{i=1}^{n}\left[\left(\frac{d \phi_{i}}{d \alpha_{j}}\right)^{2} \frac{d\left(\rho D_{i}\right)}{d \alpha_{j}}\left(\frac{d \alpha_{j}}{d x}\right)^{2}+\frac{d \phi_{i}}{d \alpha_{j}} \rho D_{i} \frac{d^{2} \phi_{i}}{d \alpha_{j}^{2}}\left(\frac{d \alpha_{j}}{d x}\right)^{2}+\left(\frac{d \phi_{i}}{d \alpha_{j}}\right)^{2} \rho D_{i} \frac{d^{2} \alpha_{j}}{d x^{2}}\right] / \\
& \sum_{i=1}^{n}\left(\frac{d \phi_{i}}{d \alpha_{j}}\right)^{2}-\rho\left(\boldsymbol{\xi}_{j}, w\right)=0, \quad j=1, \ldots, n_{c}
\end{aligned}
$$

Apart from the controlling variables we have to calculate the enthalpy in the domain. As we use unit Lewis numbers, the enthalpy does not change in the downstream part of the burner. Therefore, the enthalpy is known in the entire domain downstream of the burner, if it is known on the burner edge. As we are considering a one-dimensional flame, the flame is only cooled at the burner edge. The enthalpy on the burner edge is obtained by the specific mole numbers of the species on the burner edge, which are known as function of the controlling variables and the temperature on the burner, which is fixed.

Equations (15) are non-linear in $\alpha_{j}$. Before we can solve the equations numerically the non-linear term $\left(d \alpha_{j} / d x\right)^{2}$ is linearized by writing it as $\left(d \alpha_{j} / d x\right)_{k-1}\left(d \alpha_{j} / d x\right)_{k}$, where $k$ denotes the iteration index. The remaining equations are discretized using the finite-volume method of Thiart (1990).

Starting with a first estimate for the solution, the numerical procedure consists of the following steps. First, the contributions to one differential equation are calculated. Then the equation is linearized, discretized and solved by the use of a tridiagonal solver. This procedure is repeated for the equations of the other controlling solver. This procedure is repeated for the equations of the other controlling variables. The enthalpy is updated finally. It is expected that the solution method can be improved considerably by using an implicit method in which the equations of all variables are solved simultaneously. Further improvement can be established by using a local grid refinement method on the manifold. Here, we use equidistant grids for the time being for reasons of simplicity. 


\section{RESULTS}

First we apply the above described method on flat flames using one-step reduced schemes using reaction schemes without and with $\mathrm{HO}_{2}$, denoted as System I and II, respectively. The reaction schemes are shown in Appendix B. For these reduced schemes we used $\phi_{\mathrm{H}_{2} \mathrm{O}}$ as controlling variable. The results are compared with results of calculations using the full reaction system. We use unit Lewis numbers $\left(L e_{i}=1\right)$ and constant and for all species equal specific heat $c_{p_{i}}=c_{p}$. These approximations are not essential for the reduction method and are introduced for simplicity. The one-step reduced scheme of System $I$ is applied to burner-stabilized flame calculations with several equivalence ratios $\boldsymbol{\Phi}$ and mass flow rates $\dot{M}$.

In Figure 1 we show one example of the results of the full scheme and reduced mechanism. The temperature at a distance of $1.0 \mathrm{~cm}$ down-stream of the burner is shown in Figure 2 for all full scheme and reduced calculations. Considering these figures we may conclude that the one-step reduced scheme of System I gives quite accurate results compared with full scheme calculations. Moreover, it can be seen that the differences between reduced and full scheme computations increase for increasing $\boldsymbol{\Phi}$ values.

To apply the one-step reduced scheme of System II to burner-stabilized flame calculations, the flow velocity must be considerably lower than the adiabatic burning velocity, otherwise the flame will blow off. This agrees with results obtained before

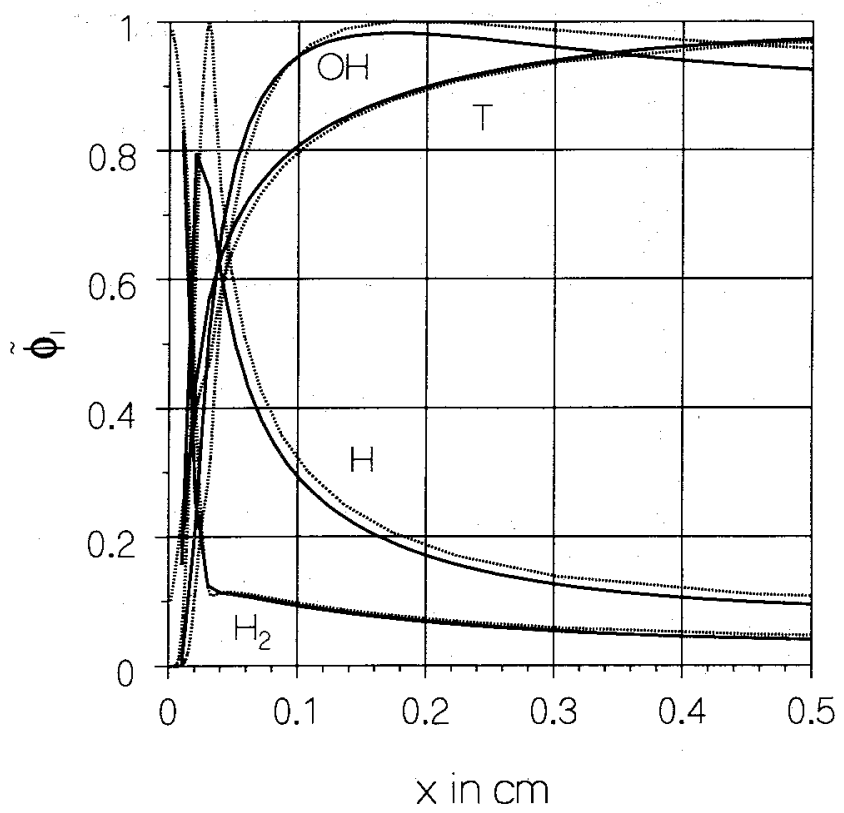

FIGURE 1: Profiles of species using full (lines) and one-step reduced mechanism (dashed lines) applied to burner-stabilized flames with an equivalence ratio $\phi=0.9$ and mass flow rate $\dot{M}=0.10 \mathrm{~g} /\left(\mathrm{cm}^{2}\right.$ s), for reaction System 1. The maximum values (scaled to 1) are: $\mathrm{H}: 8.110^{-2}, \mathrm{OH}: 2.410^{-2}, \mathrm{H}_{2}: 2.610^{-1}$ and $T: 2610$. 


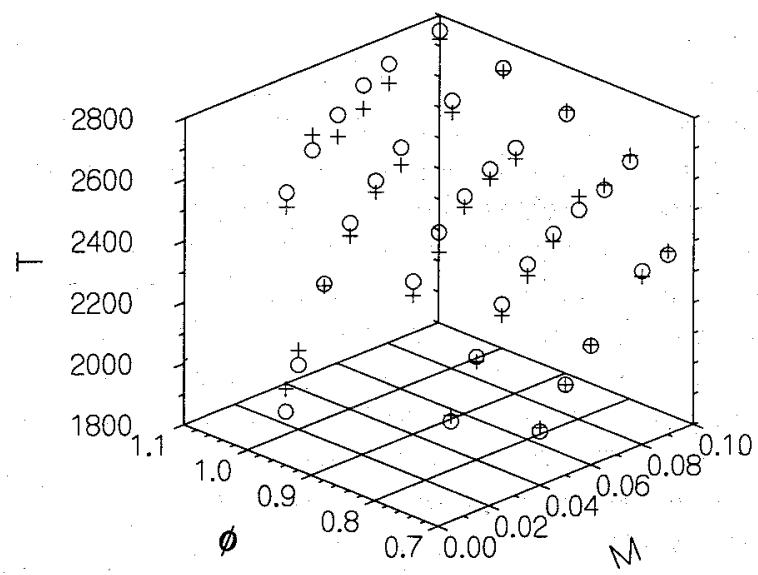

FIGURE 2 Comparison of flame temperature at a height of $1.0 \mathrm{~cm}$ down-stream of the burner between one-step reduced ( + ) and full scheme (o) calculations, as function of equivalence ratio $\phi$ and mass flow rate $\dot{M}$, for reaction System I.

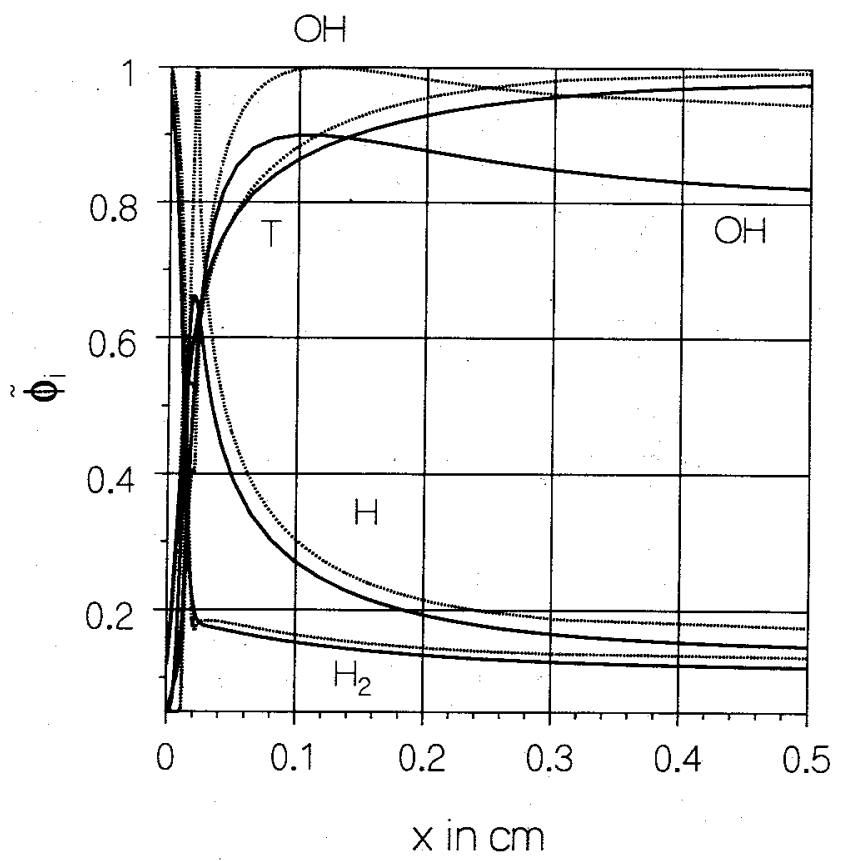

FIGURE 3 Profiles of species using full (lines) and one-step reduced mechanism (dashed lines) applied to burner-stabilized flames with an equivalence ratio $\Phi=1.0$ and mass flow rate $\dot{M}=0.14 \mathrm{~g} /\left(\mathrm{cm}^{2} \mathrm{~s}\right)$, for reaction System II. The maximum values (scaled to 1) are: $\mathrm{H}: 8.510^{-2}, \mathrm{OH}: 2.410^{-2} \mathrm{H}_{2}: 2.810^{-2}$ and $T: 2710$. 
(Eggels, 1995) for adiabatic flames, where it is found that the adiabatic burning velocity of the one-step reduced scheme is considerably lower than the burning velocity of the full system, in case of System II.

Results of the one-step reduced scheme calculations using a mass flow rate of $0.14 \mathrm{~g} /\left(\mathrm{cm}^{2} \mathrm{~s}\right)$ are presented in Figure 3. The adiabatic mass burning rate for System II is equal to $0.193 \mathrm{~g} /\left(\mathrm{cm}^{2} \mathrm{~s}\right)$. It can be seen that the profiles of the main species are predicted surprisingly well. The results of the radicals show larger differences. The scheme predicts the flame structure still quite well, because the composition is predicted well in the high temperature region.

As the one-step reduced scheme of System II can not be used for flow velocities close to the burning velocity, a two-step reduced scheme is used, to study whether this gives improvements. As controlling variables we use the specific mole numbers of $\mathrm{H}_{2} \mathrm{O}$ and $\mathrm{H}_{2}$. These controlling variables increase or decrease monotonously with $x$, as was required when Equation (14) was derived.

The calculation using the two-dimensional scheme is performed for a mass flow rate $\dot{M}=0.19 \mathrm{~g} /\left(\mathrm{cm}^{2} \mathrm{~s}\right)$, which is close to the adiabatic mass burning rate. The results are shown in Figures 4 and 5. Note that flames using the one-step reduced scheme will blow off at this flow rate. The two-step reduced scheme, however, gives stable flames. The results show that the specific mole numbers of the species are predicted well. The main difference between reduced and full scheme calculations is that the stand-off distance of the computation with the reduced scheme is larger. The specific mole numbers show more deviations in the last part of the flame. This is mainly caused by the relatively large

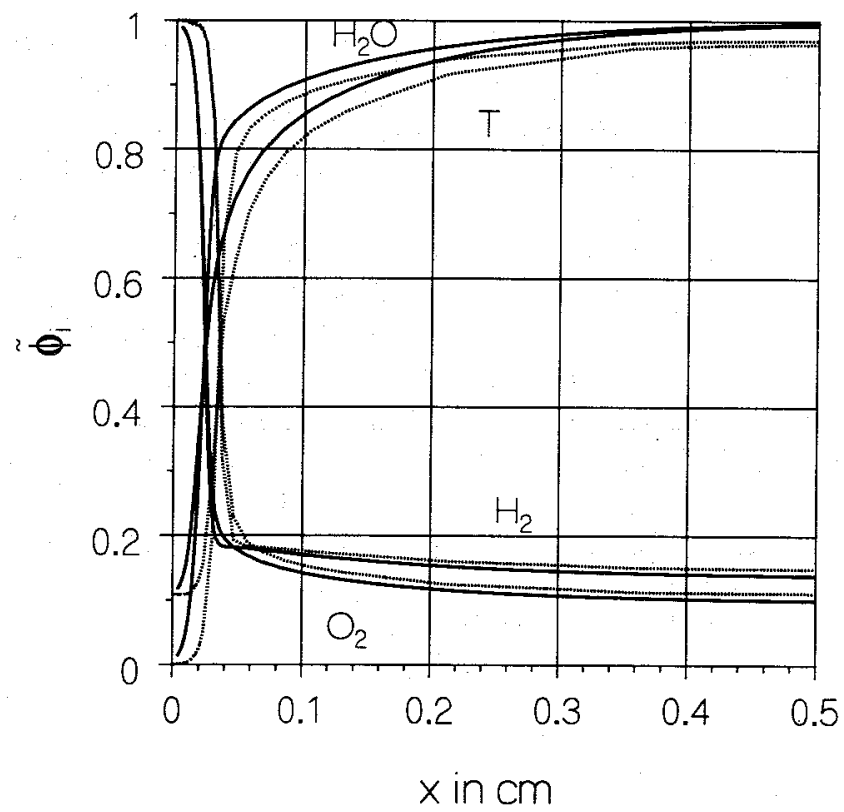

FIGURE 4 Comparison of full (lines) and two- step reduced scheme calculations (dashed lines) with an equivalence ratio of $\Phi=1.0$ and mass flow rate of $\dot{M}=0.19 \mathrm{~g} /\left(\mathrm{cm}^{2} \mathrm{~s}\right)$, for reaction System II. The maximum values (scaled to 1 ) are: $\mathrm{O}_{2}: 1.510^{-1}, \mathrm{H}_{2}: 3.010^{-1}, \mathrm{H}_{2} \mathrm{O}: 2.710^{-1}$ and $T: 2750$. 


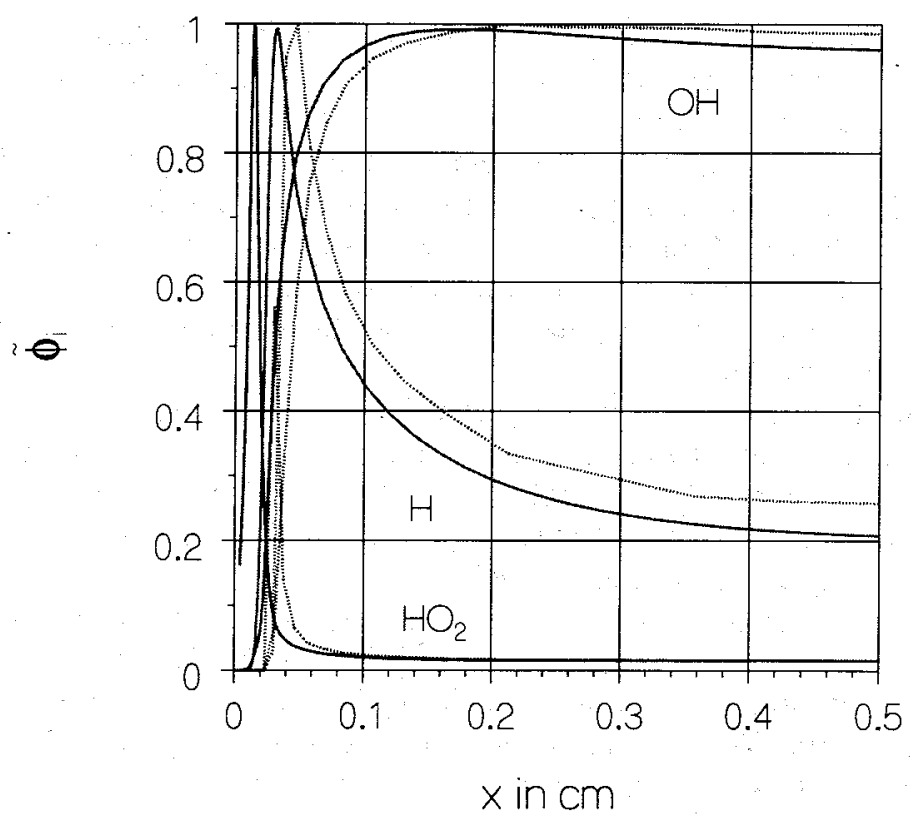

FIGURE 5 Comparison of full (lines) and two- step reduced scheme calculations (dashed lines) with an equivalence ratio of $\boldsymbol{\phi}=1.0$ and mass flow rate of $\dot{M}=0.19 \mathrm{~g} /\left(\mathrm{cm}^{2} \mathrm{~s}\right)$, for reaction System II. The maximum values (scaled to 1) are: $\mathrm{H}: 6.710^{-2}, \mathrm{OH}: 2.710^{-2}$ and $\mathrm{HO}_{2}: 2.410^{-4}$.

grid distance on the manifold there. Furthermore, it appears that an explicit solution method (solving the differential equations for the controlling variables decoupled) can be used, due to the fact that the degree of stiffness is reduced by using the reduction method. It is, however, expected that an implicit method (solving all variables simultaneously) will be faster.

A few subjects will be studied in more detail in the near future. First of all a problem that arises during the application of the two-step reduced scheme is that areas on the manifold are reached where no accurate interpolation can be made. This is related with the fact that not for all values of the controlling variables manifold compositions are physically possible (the specific mole numbers may not be negative, the temperature of the mixture may not be lower than the temperature of the unburned mixture and the manifold composition has to obey the element conservation equations). Therefore, it might occur that there are not enough grid points surrounding the interpolation point to make an accurate interpolation possible. Furthermore, to obtain more physically realistic results non unit Lewis numbers effects should be incorporated in future studies.

\section{CONCLUSIONS}

A reduction technique, which assumes fast reaction groups in steady-state, is applied to reduce hydrogen/air reaction mechanisms. The fast reaction groups are obtained with 
the use of an eigenvector analysis of the chemical source term. The one- and two-step reduced schemes are applied to flat burner-stabilized hydrogen/air flames and the results are compared with full scheme calculations. The one-step reduced scheme applied to a reaction scheme that does not include $\mathrm{HO}_{2}$, gives quite accurate results. If $\mathrm{HO}_{2}$ is taken into consideration the one-step reduced scheme can only be used for low mass flow rates. The flame will blow off otherwise. For small mass flow rates, the one-step reduced scheme still gives quite accurate results for the flame structure. For a large mass flow rate a two-step reduced scheme is used, which gives appropriate results.

\section{APPENDIX A: PROJECTION METHOD}

In this section two projection methods are presented to transform the differential equations for the species (14) to differential equations for the controlling variables (15). The projection method used in this paper makes use of the tangential direction vectors of the manifold. A method based on the eigenvectors can also be used. We will show that the projection of the convection and the source term are identical for both methods. Moreover, the projections of these terms are identical to the corresponding terms of the initial Equations (11) of those species which are used as controlling variables. This means that only the diffusion term in the reduced scheme equations, differs from the diffusion term of Equation (11). The projection of the diffusion term is considered in more detail in the last section.

\section{Projection Method Based on Tangential Direction Vectors}

For a one-dimensional geometry (flat flame) there is only one direction vector, as the combustion process is a one-dimensional path on the manifold. Therefore, we may write:

$$
\frac{d \phi}{d \alpha_{1}}=\frac{d \phi}{d \alpha_{2}} \frac{d \alpha_{2}}{d \alpha_{1}}
$$

Note that $d \phi / d \alpha_{i}$ denotes the total derivative (see Eq. (12)). The projection operator which transforms the differential equations of the species into differential equations for the controlling variables is given by the $n \times n_{c}$ matrix $\mathbf{P}^{\prime}$ :

$$
\mathbf{P}^{\prime}=\left(\begin{array}{ccc}
- & \boldsymbol{p}_{1} & - \\
\vdots & \\
- & \boldsymbol{p}_{n_{c}} & -
\end{array}\right),
$$

with $\boldsymbol{p}_{i}$ given by:

$$
\boldsymbol{p}_{i}=\frac{d \boldsymbol{\phi}}{d \alpha_{i}} /\left|\frac{d \phi}{d \alpha_{i}}\right|^{2} .
$$

This is an orthogonal projection, which is also used by Pope and Maas (1993) to project the differential equations of the species on Trajectory Generated Low-dimensional 
Manifolds. We will show that the projected convection and chemical source terms are identical to the terms in the initial species Equation (11) for the controlling variable.

First, the convection term $\dot{M}\left(\left(d \phi_{i} / d \alpha_{j}\right)\left(d \alpha_{j} / d x\right)\right)$ is considered. The $j^{\text {th }}$ component of the projection of the tangential direction vector $d \phi_{i} / d \alpha_{j}$ is given by:

$$
\left(\mathbf{P}^{\prime} \frac{d \boldsymbol{\phi}}{d \alpha_{i}}\right)_{j}=\left(\frac{d \boldsymbol{\phi}}{d \alpha_{i}}, \frac{d \boldsymbol{\phi}}{d \alpha_{j}}\right) /\left|\frac{d \boldsymbol{\phi}}{d \alpha_{j}}\right|^{2}=\left(\frac{d \boldsymbol{\phi}}{d \alpha_{j}}, \frac{d \boldsymbol{\phi}}{d \alpha_{j}}\right) /\left|\frac{d \boldsymbol{\phi}}{d \alpha_{j}}\right|^{2} \frac{d \alpha_{j}}{d \alpha_{i}}=\frac{d \alpha_{j}}{d \alpha_{i}},
$$

leading to:

$$
\mathbf{P}^{\prime}\left(\begin{array}{c}
\frac{d \phi_{1}}{d \alpha_{i}} \\
\vdots \\
\frac{d \phi_{n}}{d \alpha_{i}}
\end{array}\right)=\left(\begin{array}{c}
\frac{d \alpha_{1}}{d \alpha_{i}} \\
\vdots \\
\frac{d \alpha_{n_{c}}}{d \alpha_{i}}
\end{array}\right) .
$$

When we apply this projection operator on the convection term $\dot{M}(d \phi / d x)$, we obtain for the $j^{\text {th }}$ controlling variable:

$$
\dot{M}\left(\mathbf{P}^{\prime} \frac{d \boldsymbol{\phi}}{d \alpha_{i}}\right)_{j} \frac{d \alpha_{i}}{d x}=\dot{M} \frac{d \alpha_{j}}{d x}
$$

which is equal to the convective term in the differential equation of $\phi_{i}$ corresponding to controlling variable $\alpha_{j}=\phi_{i}$.

Now, let us consider the projection of the source term $w$. As the eigenvectors $s_{i}$ form a basis of composition space, the source term may be written as:

$$
\boldsymbol{w}=\sum_{i=1}^{n} \beta_{i} \boldsymbol{s}_{i} .
$$

Using the definition of the manifold $\left(s_{i}^{L}, w\right)=0$, for $i=n_{c}+n_{e}+1, \ldots, n$ and $\left(\boldsymbol{s}_{i}^{L}, \boldsymbol{s}_{j}\right)=\delta_{i j}$, where $\delta_{i j}$ denotes the kronecker delta, we get:

$$
\boldsymbol{w}=\sum_{i=1}^{n_{c}} \beta_{i} \boldsymbol{s}_{i}
$$

The tangential direction vectors are parallel to the manifold. The manifold, however, is also spanned by the first $n_{c}$ eigenvectors so that we write:

$$
\frac{d \boldsymbol{\phi}}{d \alpha_{i}}=\sum_{j=1}^{n_{c}} a_{i j} \mathbf{s}_{j} .
$$

From Equations (23) and (24) it can be seen that the number of degrees of freedom is $n_{c}$. The equation used to define the manifold and time scales is given by:

$$
\frac{d \boldsymbol{\phi}}{d \alpha_{i}} \frac{d \alpha_{i}}{d t}=\boldsymbol{w}
$$

Although these are $n$ equations, the vectors $d \phi / d \alpha_{i}$ and $w$ are restricted to a $n_{c}$ dimensional subspace, so there are only $n_{c}$ degrees of freedom. Therefore, we may conclude from (25) that the tangential direction vectors and the source term are 
parallel, so that the source term may be written as:

$$
w=\frac{d \phi}{d \alpha_{j}}\left(\xi_{j}, w\right)
$$

With the use of (19) it follows easily that:

$$
\mathbf{P}^{\prime} w=\left(\left(\xi_{1}, w\right), \ldots,\left(\xi_{n_{c}}, w\right)\right)^{T},
$$

which is again the source term of the differential equation for the species corresponding to the controlling variable.

\section{Eigenvector Projection}

The manifold is defined by steady-state assumptions for fast reaction groups, which are found by the use of an eigenvector analysis. In the basis of eigenvectors the eigenvalues are ordered from large to small real parts (Note that most real parts are negative). Therefore, the reaction groups are ordered from slow to fast. For the reduced scheme only slow reaction groups will be considered. To obtain the evolution equations for these slow processes only, we make use of the following filtering $(n \times n)$ matrix $\mathbf{F}^{\prime}$, defined in the basis of eigenvectors:

$$
\mathbf{F}^{\prime}=\left(\begin{array}{ll}
\text { I } & 0 \\
\mathbf{0} & 0
\end{array}\right),
$$

with I the $n_{c} \times n_{c}$ identity matrix. All other matrix elements are zero. In the original basis this becomes:

$$
\mathbf{F}=\mathbf{S}\left(\begin{array}{ll}
1 & 0 \\
0 & 0
\end{array}\right) \mathbf{S}^{-1}
$$

The next step transforms the differential equations into differential equations for the controlling variables. This gives the following transformation matrix:

$$
\mathbf{P}=\mathbf{K S}\left(\begin{array}{ll}
\mathbf{I} & 0 \\
\mathbf{0} & 0
\end{array}\right) \mathbf{S}^{-1},
$$

where $\mathbf{K}$ is a $n \times n_{c}$ matrix, so that $\mathbf{P}$ is also a $n \times n_{c}$ matrix. Since the controlling variables are given by $\alpha_{i}=\left(\xi_{i}^{T}, \phi\right)$, the elements of $\mathbf{K}$ are given by $k_{i j}=\xi_{i j}$. For example, if the specific mole numbers of species with index 5 and index $1\left(\alpha_{1}=\phi_{5}\right.$ and $\left.\alpha_{2}=\phi_{1}\right)$ are used as controlling variables in a 2-D $\left(n_{c}=2\right)$ reduced scheme and the number of species is $n=7$, the matrix $\mathbf{K}$ has the following form:

$$
\mathbf{K}=\left(\begin{array}{lllllll}
0 & 0 & 0 & 0 & 1 & 0 & 0 \\
1 & 0 & 0 & 0 & 0 & 0 & 0
\end{array}\right)
$$

Let us now consider the projection of the source term $F w$ in more detail. For $w-F w$ we may write:

$$
(\mathbf{I}-\mathbf{F}) \boldsymbol{w}=\mathbf{S}\left(\mathbf{I}-\left(\begin{array}{ll}
\mathbf{I} & 0 \\
0 & 0
\end{array}\right)\right) \mathbf{S}^{-1} w=\mathbf{S}\left(\begin{array}{ll}
\mathbf{0} & 0 \\
0 & \mathbf{I}
\end{array}\right) \mathbf{S}^{-1} \boldsymbol{w}=0
$$


Since $\left(s_{i}^{L}, w\right)=0$ for $i=n_{c}+n_{e}+1, \ldots, n$ by definition of the manifold. We have found that $\mathbf{w}$ is not effected by the projection $\mathbf{F}$ :

$$
\mathbf{F} w=w .
$$

Applying the projection and transformation on the source term gives:

$$
\mathbf{P} w=\left(\left(\xi_{i}, w\right), \ldots,\left(\xi_{n_{c}}, w\right)\right)^{T},
$$

leading to the same result as the projection method based on the tangential direction vectors (Eq. 27).

Now, we consider the convection term $\dot{M}\left(d \phi / d \alpha_{i}\right)\left(d \alpha_{i} / d x\right)$. Using (24) and $\left(s_{i}^{L}, s_{j}\right)=\delta_{i j}$ gives:

$$
\mathbf{F} \frac{d \phi}{d \alpha}=\frac{d \phi}{d \alpha}
$$

and further we get:

$$
\mathbf{P}^{\prime}\left(\begin{array}{c}
\frac{d \phi_{1}}{d \alpha_{i}} \\
\vdots \\
\frac{d \phi_{n}}{d \alpha_{i}}
\end{array}\right)=\left(\begin{array}{c}
\frac{d \alpha_{1}}{d \alpha_{i}} \\
\vdots \\
\frac{d \alpha_{n_{c}}}{d \alpha_{i}}
\end{array}\right)
$$

This agrees with the result we have found for the other projection method (19). From this we may conclude that $\mathbf{P}$ and $\mathbf{P}^{\prime}$ give identical results for the convective and source terms in the differential Equation (11).

\section{Projection of the Diffusion Term}

In this next section we turn to the projection of the diffusive terms. For a stationary flat flame the differential equations are given by:

$$
\dot{M} \frac{d \phi}{d \alpha_{i}} \frac{d \alpha_{i}}{d x}-\pi=w
$$

where $\pi$ represents the diffusion term, with $\pi_{i}=d / d x\left(\rho D_{i}\left(d \phi_{i} / d x\right)\right)$. Projection of this equation on the manifold gives:

$$
\dot{M} \frac{d \alpha_{i}}{d x}-\mathbf{P} \pi=\left(\xi_{i}, w\right)^{T}
$$

As shown in Equation (15) the diffusion term may be written as:

$$
\pi_{i}=-\frac{d\left(\rho D_{i}\right)}{d \alpha_{j}} \frac{d \phi_{i}}{d \alpha_{j}}\left(\frac{d \alpha_{j}}{d x}\right)^{2}-\rho D_{i} \frac{d^{2} \phi_{i}}{d \alpha_{j}^{2}}\left(\frac{d \alpha_{j}}{d x}\right)^{2}-\rho D_{i} \frac{d \phi_{i}}{d \alpha_{j}} \frac{d^{2} \alpha_{j}}{d x^{2}}
$$


For equal diffusion coefficients $\left(D_{i}=D\right)$, the first and the third terms of Equation (39) are parallel to $d \phi / d \alpha_{j}$. Note that terms parallel to $d \phi / d \alpha_{j}$ give identical results for both projection methods. The only term which is not parallel to the manifold in case of identical $D_{i}$ is the second term of Equation (39). Differences between the two mentioned projection methods arise if this term is large. It is expected, however, that the differences between the projection methods are small, because of the fact that processes are much faster outside the manifold than on the manifold. This implies that process will first move towards the manifold rapidly and will propagate subsequently on the manifold much slower. In fact, $\mathbf{P}$ and $\mathbf{P}^{\prime}$ both project perpendicular to the manifold in case of infinitely fast processes outside the manifold. Only in situations where the time scale difference between processes outside the manifold and processes on the manifold are small, the projection method based on the tangential direction vectors will be less accurate. Then, however, the reduction method in itself is not accurate: the eigenvectors are not accurate because diffusion effects are of importance in that case.

\section{APPENDIX B: USED REACTION SCHEMES}

The reaction rates of reaction $i$ are given by the Arrhenius expression: $A_{i} T^{\beta_{i}} \exp \left(-E_{i} / R T\right)$, where $A_{i}$ and $\beta_{i}$ are reaction constants, $E_{i}$ the activation energy, $R$ the universal gas constant and $T$ the temperature. The coefficients $A_{i}, \beta_{i}$ and $E_{i}$ are successively given after the reactions $\left(A_{i}\right.$ in $\mathrm{cm} / \mathrm{mol} / \mathrm{s} ; E_{i}$ in $\left.\mathrm{KJ} / \mathrm{mol}\right)$. Used collision efficiencies are: $f_{\mathrm{H}_{2}}=1.00, f_{\mathrm{O}_{2}}=0.35, f_{\mathrm{H}_{2} \mathrm{O}}=6.50, f_{\mathrm{N}_{2}}=0.50, f_{\mathrm{CO}}=1.50$, $f_{\mathrm{CO}_{2}}=1.50$.

\section{System I}

This reaction scheme includes the following species: $\mathrm{O}_{2}, \mathrm{H}, \mathrm{OH}, \mathrm{O}, \mathrm{H}_{2}, \mathrm{H}_{2} \mathrm{O}$ and $\mathrm{N}_{2}$ The following reactions are used:

$\begin{array}{llcc}\mathrm{H}+\mathrm{O}_{2}=\mathrm{OH}+\mathrm{O} & 2.0010^{14} & 0.0 & 70.3 \\ \mathrm{OH}+\mathrm{O}=\mathrm{H}+\mathrm{O}_{2} & 1.4610^{13} & 0.0 & 2.08 \\ \mathrm{O}+\mathrm{H}_{2}=\mathrm{OH}+\mathrm{H} & 5.0610^{4} & 2.67 & 26.3 \\ \mathrm{OH}+\mathrm{H}=\mathrm{O}+\mathrm{H}_{2} & 2.2410^{4} & 2.67 & 18.4 \\ \mathrm{H}_{2}+\mathrm{OH}=\mathrm{H}_{2} \mathrm{O}+\mathrm{H} & 1.0010^{8} & 1.6 & 13.8 \\ \mathrm{H}_{2} \mathrm{O}+\mathrm{H}=\mathrm{H}_{2}+\mathrm{OH} & 4.4510^{8} & 1.6 & 77.13 \\ \mathrm{OH}+\mathrm{OH}=\mathrm{O}+\mathrm{H}_{2} \mathrm{O} & 1.5010^{9} & 1.14 & 0.42 \\ \mathrm{O}+\mathrm{H}_{2} \mathrm{O}=\mathrm{OH}+\mathrm{OH} & 1.5110^{10} & 1.14 & 71.64 \\ \mathrm{H}+\mathrm{H}+\mathrm{M}=\mathrm{H}_{2}+\mathrm{M} & 1.8010^{18} & -1.00 & 0.0 \\ \mathrm{H}_{2}+\mathrm{M}=\mathrm{H}+\mathrm{H}+\mathrm{M} & 6.9910^{18} & -1.00 & 436.08 \\ \mathrm{OH}+\mathrm{H}+\mathrm{M}=\mathrm{H}_{2} \mathrm{O}+\mathrm{M} & 2.2010^{22} & -2.00 & 0.0 \\ \mathrm{H}_{2} \mathrm{O}+\mathrm{M}=\mathrm{OH}+\mathrm{H}+\mathrm{M} & 3.8010^{23} & -2.00 & 499.41 \\ \mathrm{O}+\mathrm{O}+\mathrm{M}=\mathrm{O}_{2}+\mathrm{M} & 2.9010^{17} & -1.00 & 0.0 \\ \mathrm{O}_{2}+\mathrm{M}=\mathrm{O}+\mathrm{O}+\mathrm{M} & 6.8110^{18} & -1.00 & 496.41\end{array}$




\section{System $I I$}

Reactions and species of System I, extended with species $\mathrm{HO}_{2}$ and the following reactions.

$\begin{array}{lccc}\mathrm{H}+\mathrm{O}_{2}+\mathrm{M}=\mathrm{HO}_{2}+\mathrm{M} & 2.3010^{18} & -0.80 & 0.0 \\ \mathrm{HO}_{2}+\mathrm{M}=\mathrm{H}+\mathrm{O}_{2}+\mathrm{M} & 2.2610^{18} & -0.80 & 195.88 \\ \mathrm{HO}_{2}+\mathrm{H}=\mathrm{OH}+\mathrm{OH} & 1.5010^{14} & 0.00 & 4.2 \\ \mathrm{OH}+\mathrm{OH}=\mathrm{HO}_{2}+\mathrm{H} & 1.3310^{13} & 0.00 & 168.3 \\ \mathrm{HO}_{2}+\mathrm{H}=\mathrm{H}_{2}+\mathrm{O}_{2} & 2.5010^{13} & 0.00 & 2.9 \\ \mathrm{H}_{2}+\mathrm{O}_{2}=\mathrm{HO}_{2}+\mathrm{H} & 6.8410^{13} & 0.00 & 243.10 \\ \mathrm{HO}_{2}+\mathrm{H}=\mathrm{H}_{2} \mathrm{O}+\mathrm{O} & 3.0010^{13} & 0.00 & 7.2 \\ \mathrm{H}_{2} \mathrm{O}+\mathrm{O}=\mathrm{HO}_{2}+\mathrm{H} & 2.6710^{13} & 0.00 & 242.52 \\ \mathrm{HO}+\mathrm{O}=\mathrm{OH}+\mathrm{O}_{2} & 1.8010^{13} & 0.00 & -1.7 \\ \mathrm{OH}+\mathrm{O}_{2}=\mathrm{HO}_{2}+\mathrm{O} & 2.1810^{13} & 0.00 & 230.6 \\ \mathrm{HO}_{2}+\mathrm{OH}=\mathrm{H}_{2} \mathrm{O}+\mathrm{O}_{2} & 6.0010^{13} & 0.00 & 0.0 \\ \mathrm{H}_{2} \mathrm{O}+\mathrm{O}_{2}=\mathrm{HO}_{2}+\mathrm{OH} & 7.3110^{14} & 0.00 & 303.53\end{array}$

\section{ACKNOWLEDGEMENTS}

The support of Gastec N.V. and NOVEM, The Netherlands, is gratefully acknowledged.

\section{REFERENCES}

Eggels, R. L. G. M. and Goey de, L. P. H. (1995). Mathematically Reduced Reaction Mechanisms Applied to Adiabatic Flat Hydrogen/Air Flames. Combust. and Flame, 100, 559.

Goussis, D. A. and Lam, S. H. (1992). Twenty-Fourth Symposium (International) on Combustion. The Combustion Institute, Pittsburgh, p. 113.

Lam, S. H. and Goussis, D. A. (1988). Twenty-Second Symposium (International) on Combustion. The Combustion Institute, Pittsburgh, p. 931.

Maas, U. and Pope, S. B. (1992). Simplifying Chemical Kinetics: Intrinsic Low-Dimensional Manifolds in Composition Space. Combust. Flame, 88, 239.

Maas, U. and Pope, S. B.(1992). Twenty-Fourth Symposium (International) on Combustion. The Combustion Institute, Pittsburgh, p. 103.

Maas, U. and Pope, S. B. (1994). Laminar Flame Calculations Using Simplified Chemical Kinetics Based on Intrinsic Low-Dimensional Manifolds. Twenty-Fifth Symposium (International) on Combustion. The Combustion Institute, Pittsburgh.

Peters, N. and Williams, F. A. (1987) The Asymptotic Structure of Stoichiometric Methane-Air Flames. Combust. Flame, 68, 185.

Peters, N. and Rogg, B. (1993). Reduced Kinetics Mechanisms for Applications in Combustion Systems, Springer.

Pope, S. B. and Maas U. Simplifying Chemical Kinetics: Trajectory-Generated Low-Dimensional Manifolds, Cornell Report FDA 93-11.

Smooke, M. D. (1991). Reduced Kinetic Mechanisms and Asymptotic Approximations for Methane-Air Flames. Lecture Notes in Physics 384, Springer.

Thiart, G. D. (1990). Finite Difference Scheme for the Numerical Solution of Fluid Flow and Heat Transfer Problems on Nonstaggered Grids, Numerical Heat Transfer Part B, 17, 43. 